Une prise de position de la Commission de spécialité de la Société suisse d'allergologie et d'immunologie (SSAI) sur la biorésonance et les appareils d'électroacupuncture utilisés dans le diagnostic et la thérapie d'états soi-disant allergiques

\title{
La biorésonance: un non-sens diagnostique et thérapeutique
}

B. Wüthrich ${ }^{a}$, P. C. Frei ${ }^{b}$, A. Bircherc, C. Hauser, W. Pichler ${ }^{e}$, P. SchmidGrendelmeierf, F. Spertini ${ }^{g}$, D. Olgiati ${ }^{h}$, U. Müller $^{i}$

a Praxis für Allergologie und Dermatologie, Spital Zollikerberg

b Chemin de Mourat 73, 1095 Lutry

c Allergologische Poliklinik, Dermatologische Universitätsklinik Basel

d Unité d'Allergologie, Service d'Immunologie et d'Allergologie et Service de Dermatologie, Genève

e Klinik für Rheumatologie und Klinische Immunologie/ Allergologie, Inselspital Bern

f Allergiestation, Dermatologische Universitätsklinik, Zürich

g Service d'immunologie et d'allergologie, CHUV, Lausanne

h Präsidentin der Fachkommission der SGAI, Praxis für Innere Medizin und Allergologie, Delsberg

i Präsident der SGAI, Medizinische Klinik, Zieglerspital, Bern
Correspondance:

Pr Dr Brunello Wüthrich Spital Zollikerberg

Trichtenhauserstrasse 20

CH-8125 Zollikerberg

E-mail: sgai@bluewin.ch;

brunello.wuethrich@

spitalzollikerberg.ch

\section{Introduction}

La Commission de spécialité de la Société suisse d'allergologie et d'immunologie (SSAI) a publié dans le Bulletin des médecins suisses, en 1995 déjà, une prise de position sur des procédés paramédicaux appliqués à des affections allergiques, et en particulier sur la biorésonance [1]. Dans un article consacré à la «Good Allergy Practice» [2], elle a pris position sur les procédés de médecine alternative appliqués à l'allergologie, de la façon suivante: «Pour aucunes des méthodes de la médecine alternative, il n'existe de valeur diagnostique ni d'effet thérapeutique qui soit significativement démontré.»

Il existe aussi des prises de positions négatives sur la biorésonance de la part de diverses sociétés européennes de la discipline [3-5] et des articles qui jugent ces procédés comme non scientifiques [6-10]. Pourtant, une publicité intense continue à se faire en faveur de l'introduction de telles méthodes appliquées à des affections allergiques («Effacer l'allergie avec la biorésonance»), à l'aide d'appareils (Bicom, Mora, MitoSan ou Vegatest I et II) [11].

Ni la biorésonance ni les méthodes d'électroacupuncture ne sont remboursées par l'assurance-maladie obligatoire. Elles ne font ainsi pas l'objet de la Décision du Département fédéral de l'intérieur sur les médecines complémentaires du juin 2005. Cependant, les appareils de biorésonance et d'électro-acupuncture ne sont pas utilisés seulement par des médecins adeptes de médecines alternatives, mais aussi par des généralistes, des dentistes, des podologues, des kinésiologues, des chiropraticiens, des droguistes, des naturopathes, etc. [12]. Dans le Canton de Zurich, par exemple, de tels procédés sont classifiés ( 3 , lettre $\mathrm{K}$ de l'Ordonnance sur les professions de la santé) comme «méthodes dites externes, non dangereuses et non scientifiques», un peu comme l'imposition des mains ou la prière, et ne sont ainsi pas soumis à autorisation [13]. En conséquence, il n'existe, pour l'autorité sanitaire, aucune obligation de surveillance.
Les caisses-maladie, elles offrent la couverture de ces procédés au travers d'assurances complémentaires, qu'elles s'efforcent d'introduire auprès d'éventuels clients, à l'aide de publicité [14]. Une condition pour l'introduction de nouvelles méthodes diagnostiques et thérapeutiques devrait être un contrôle approfondi des appareils utilisés et la prise en charge de prestations par des assurances complémentaires devrait être faite avec le même soin que pour la médecine conventionnelle: c'est-à-dire que les prestations ne devraient être remboursables qu'une fois faite la preuve de leur efficacité. De cette façon seulement, le patient pourrait être protégé contre des procédés inutiles, trompeurs, ou parfois même dangereux.

Les soi-disant succès de traitements sans base rationnelle reposent sur l'effet placebo ou s'expliquent par l'évolution naturelle de l'affection traitée. Il n'est pas acceptable que, en une époque d'économie des ressources, les caisses-maladie veuillent rembourser, par l'assurance complémentaire, des prestations non contrôlées. Une telle attitude est défavorable à la médecine conventionnelle comme à la médecine complémentaire sérieuse.

Ces procédés et leurs appareils sont brièvement décrits ci-après. Nous renvoyons également aux prises de positions et revues citées [1-10] et aux pages Web sur internet: www. quackwatch.org et www.allergycapital.com.au sous «alternative methods».

\section{Biorésonance et multirésonance}

A la base de la biorésonance, on postule que l'être humain et les allergènes environnants émettent un spectre vibratoire ultrafin, qui n'est pas démontrable par les méthodes de mesure physiques conventionnelles [15, 16]. Un cylindre métallique que le sujet tient dans ses mains est utilisé comme électrode de sortie, tandis que les «vibrations» sont dérivées dans l'appareil à l'aide d'un câble électrique non blindé et de prises bananes. 
L'examen de la méthode par le physicien F. Cap a montré que les bases physiques de la biorésonance ne sont pas correctes [17]. Ces résultats ont été confirmés par d'autres physiciens et ingénieurs [18].

Le patient joue le rôle d'antenne recevant toutes sortes d'ondes électromagnétiques: parasites du réseau et d'appareils électriques, signaux radar ou du téléphone, de la radio ou de la télévision. L'appareil de biorésonance n'est pas en mesure d'enregistrer des «vibrations non démontrables par les méthodes conventionnelles». Il produit seulement un ronronnement électronique, dont quelques vibrations sont éliminées à l'aide d'un filtre.

Des études cliniques ont montré que la biorésonance ne permet ni le diagnostic ni la thérapie d'affections allergiques, telles la pollinose ou la dermatite atopique [19-21]. Ces résultats sont aussi confirmés par l'expérience de divers centres d'allergologie en Suisse, en Allemagne, en Autriche et en Italie, entre autres, dans lesquels des patients avec des allergies soi-disant «effacées» demandaient à nouveau conseil [1, 22-24].

\section{Electro-acupuncture d'après le Dr Voll et diagnostic fonctionnel bio-électrique} Cette variante de l'acupuncture classique a été introduite par un médecin allemand, le Dr Voll. Il s'agit d'un procédé paramédical, qu'utilisent des naturopathes ou des praticiens de médecines alternatives pour le diagnostic ou la thérapie d'allergies alimentaires [25]. Dans ce test, l'examinateur mesure sur certains points d'acupuncture, à l'aide d'un instrument développé par le Dr Voll (p.ex. les appareils Vegatest ou Mora), un potentiel électrique, sur la base d'un courant fourni par une électrode de grande surface [26]. Si ce procédé est utilisé pour tester l'allergie, on intercale dans le courant entre l'électrode tenue par la main et l'instrument de mesure une ampoule contenant, par exemple, un extrait alimentaire. L'examinateur mesure simultanément, avec une électrode sélective, le potentiel de différents points d'acupuncture. Si la valeur mesurée est modifiée lorsqu'on interpose un certain allergène, on conclut que le patient est allergique à cette substance. En 1976 déjà, il fut démontré, lors d'investigations extensives, que cette méthode mesure des artéfacts [27]. Dans des prises de position plus récentes, l'électro-acupuncture d'après le Dr Voll et d'autres procédés semblables ont été jugés pseudo-scientifiques et non contrôlés $[8,9]$.

En Angleterre, un nouveau travail a été consacré à l'étude de la valeur diagnostique des tests allergiques électrodermiques (EAT) [28]. L'étude effectuée à l'aveugle a montré que les résultats de l'EAT ne corrèlent pas avec ceux des prick-tests et que l'EAT ne parvient pas à distinguer entre sujets atopiques et non atopiques. L'EAT semble très répandu: on estime qu'en Angleterre, plus de 500 appareils électrodermiques sont utilisés pour la mise en évidence d'allergies [29]. Ce test n'a jusqu'ici guère été vérifié cliniquement, et $s^{\prime}$ avère maintenant inutilisable, en tout cas pour la démonstration d'allergies.

\section{Tests de diagnostic et diètes injustifiés}

Les diagnostics posés à l'aide de ces méthodes non conventionnelles concernent le plus souvent des allergies aux additifs alimentaires, aux sucres, aux blés ou au lait de vache [30]. Les réactions allergiques aux additifs (désignés par les chiffres E sur les emballages) sont extrêmement rares, contrairement à ce que croient des associations de consommateurs ou de patients, ou encore certains media [31].

Le côté irrationnel de tels conseils diététiques apparaît, par exemple, lorsque, en cas d'allergie au blé diagnostiquée par méthode non conventionnelle, on prescrit la consommation d'épeautre. Cette céréale est une variante ancienne du blé, qui ne diffère pas allergèniquement du blé $[32,33]$ ! Le patient, qui n'a pas de vraie allergie au blé, croit avoir éliminé la céréale nuisible en consommant de l'épeautre. Dans des cas de soidisant allergie au lait de vache, diagnostiquée par ces mêmes méthodes, on prescrit du lait de chèvre ou de jument. L'allergie au lait de vache, à médiation $\mathrm{IgE}$, est pourtant provoquée le plus souvent par une sensibilisation à la caséine, laquelle n'est pas spécifique de l'espèce. Un patient allergique au lait de vache ne supporte pas non plus le lait de chèvre ou de jument [34].

Pour certains de ces thérapeutes, de telles diètes d'éliminations doivent être respectées aussi par les membres de l'entourage qui prennent les repas avec le patient: sinon des vibrations «dysharmoniques» provoquant l'allergie pourraient être transmises au patient! On recommande même des diètes de rotation [35], dans lesquelles, après un rituel de plusieurs séances, les allergies sont «effacées» par l'introduction de vibrations «harmoniques» en miroir.

\section{Opinions émises sur la biorésonance et les procédés d'électro-acupuncture}

Le Dr Stephan Barrett, responsable médical du site www.quackwatch.org [36], écrit: «In 1999, the British Advertising Standards Authority reviewed a pamphlet, which alleged that a Bio Resonance Therapy device could help people suffering from headaches, overweight, tiredness, 
bloating, irritable bowel syndrome, skin rashes, arthritis, and premenstrual tension. In May 1999, after reviewing a manual and other information about the device, the Authority concluded that the claims were unsubstantiated. Government agencies in several countries have taken enforcement actions related to EAV devices.» Et: «The devices described above are used to diagnose nonexistent health problems, select inappropriate treatment, and defraud insurance companies. The practitioners who use them are either delusional, dishonest, or both. These devices should be confiscated and the practitioners who use them should be prosecuted.»

Dans une prise de position actuelle, le Pr E. Ernst, de la chaire de médecine complémentaire à la Peninsula Medical School, Universités d'Exeter et Plymouth, note, entre autres [37]: «Using the example of bioresonance therapy, this article demonstrates how pseudo-scientific language can be used to cloud important issues. This can be seen as an attempt to present nonsense as science. Because this misleads patients and can thus endanger their health, we should find ways of minimizing this problem.»

\section{Conclusions}

Bien que les diverses prises de positions provenant des sociétés d'allergologie mettent unanimement en garde contre l'usage de procédés de biorésonance et d'électro-acupuncture, des maisons commerciales et des médecins (ou thérapeutes non médecins) persistent à pratiquer de telles méthodes. De plus, plusieurs caisses-maladie se déclarent prêtes à prendre leurs coûts en charge dans le cadre d'assurances complémentaires. Des patients crédules se retrouveront ainsi suivre des diètes ou des procédés «d'effacement» aberrants, croyant ainsi effacer une allergie qui n'existe pas.

En raison de l'absence de possibilité d'intervention légale, la SSAI s'adresse, au travers de la présente prise de position, au corps médical, aux patients et organisations de patients, ainsi qu'aux responsables politiques, media et caissesmaladie - et même aux praticiens de médecine complémentaire - en les mettant en garde contre l'usage de ces méthodes. Il est crucial que les caisses-maladie s'abstiennent aussi de soutenir ces méthodes à l'aide d'assurances complémentaires.

Les phénomènes physiques imaginés à la base de la biorésonance et de l'électro-acupuncture ont été jugés indéfendables par des physiciens. Les études cliniques contrôlées n'ont montré aucune corrélation avec les résultats des mé- thodes scientifiques reconnues et éprouvées, ni aucun effet thérapeutique. Des méthodes inefficaces non seulement augmentent inutilement les coûts de la médecine, mais peuvent aussi être dangereuses, si elles éloignent d'un vrai diagnostic des patients avec une affection potentiellement grave, comme, par exemple, l'anaphylaxie aux venins d'hyménoptères, ou aux aliments ou à certains médicaments [38-40]. La SSAI conclut ainsi que ces méthodes ne peuvent pas être classées comme non dangereuses et que la demande de reconnaissance ne doit pas être acceptée [41].

\section{Références}

1 Spezialistenkommission der Schweiz. Gesellschaft für Allergologie und Immunologie (SGAI). Allergologie und Alternativmedizin. Stellungnahme zu paramedizinischen Verfahren, speziell Bioresonanz, bei allergischen Erkrankungen. Bull Méd Suisses 1995;76:1229-32.

2 Müller U, de Weck AL, Bodmer R, Gutersohn J, Longoni S, Müllner G, Olgiati D, Pletscher M, Schweri T, Thürlimann W. Good Allergy Practice. Une prise de position de la Commission de specialité de la Société Suisse d'allergologie et d'immunologie. Bull Méd Suisses 2000;81:2324-39.

3 Dorsch W, Ring J, für die Arbeitsgruppe «Komplementärmedizin» der Deutschen Gesellschaft für Allergologie und klinische Immunologie. Komplementärmethoden oder so genannte Alternativmethoden in der Allergologie. Allergo J 2002; 11:163-70.

4 Kay AB, Lessof MH. The Royal College of Physicians Committee Clinical Immunology and Allergy: allergy. conventional and alternative concepts. Clin Exp Allergy 1992;22(Suppl 3):1-44.

5 Senna G, Lombardi C, Antonicelli C et al. Linee guida AAITO [Associazione Allergologia Immunologia Territoriali e Ospedalieri] sui test «alternativi» nella diagnostica delle allergopatie. Eur Ann Allergy Clin Immunol 2004; December(Suppl 2):1-8.

6 Dorsch W. Alternative in allergology: critical evaluation. In: Ring J, Behrendt H, Vieluf D (eds.). New Trends in Allergy IV. Berlin, Heidelberg: Springer; 1997. p. 319-29.

7 Niggemann B, Grüber C. Unproven diagnostic procedures in IgE-mediated allergic diseases. Allergy 2004;59:806-8.

8 Oepen I. «Alternative» Diagnose- und Therapieverfahren bei allergischen Krankheiten. Allergologie 1989;12:509-13.

9 Terr AI. Unconventional Theories and Unproven Methods in Allergy. In: Middleton E, Reed CE, Ellis EF, Adkinson NF, Yunginger JW, Busse WW (eds.). Allergy Principles and Practice. $4^{\text {th }}$ edition. St. Louis, MO: Mosby; 1993. p. 1767-93.

10 Wüthrich B. Unproven techniques in allergy diagnosis. J Invest Clin Immunol 2005;15:86-90.

11 www.regumed.ch. Treten Sie ein in die Welt der BICOM Bioresonanz-Therapie. 
12 Siegenthaler M. Bioresonanz - Mit Schwingungen heilen. Vista, Schweizer Gesundheits-Magazin 2005;2:58-9.

13 Gesundheitsdirektion Kanton Zürich. Stellungnahme vom 11. Mai 2005 an die Zürcher Gesellschaft für Allergologie (ZGA).

14 Bührig R. Komplementärmedizin. Atupri denkt ganzheitlich. atupri magazin 2004;2:9.

15 Brüggemann H. Bioresonanz- und MultiresonanzTherapie. Heidelberg: Karl Haug Verlag; 1990.

16 Schumacher P. Biophysikalische Therapie der Allergien (erweiterte Bioresonanztherapie). Stuttgart: Sonntag Verlag; 1994.

17 Cap F. Bemerkungen eines Physikers zur Bioresonanz. Allergologie 1995;18:253-7.

18 Mosenkis R. Examination of a Vegatest Device. www.quackwatch.org, September 11, 2001.

19 Kofler H, Ulmer H, Mechtler E, Falk M, Fritsch PO. Bioresonanz bei Pollinose. Eine vergleichende Untersuchung zur diagnostischen und therapeutischen Wertigkeit. Allergologie 1996;19:114-22.

20 Schöni M, Nikolacik W, Schöni-Affolter F. Efficacy trial of bioresonance in children with atopic dermatitis. Int Arch Allergy Immunol 1997; 112:238-46.

21 Wantke F, Stanek KW, Götz M, Jarisch R. Bioresonanz-Allergietest versus Pricktest und RAST. Allergologie 1993;16:144-5.

22 Niggemann B, Grüber C. Unkonventionelle Verfahren in der Allergologie. Kontroverse oder Alternative? Allergologie 2002;25:34-46

23 Ostendorf GM. Unkonventionelle apparative Methoden in der Allergietherapie. Allergologie 1995;18:221-7.

24 Schultze-Werninghaus G. Paramedizinische Verfahren: Bioresonanzdiagnostik und -therapie. Allergo J 1993;2:40-2.

25 Voll R. EAV Electro-acupuncture according to Voll. Bioenergetic diagnostics and therapy on the basis of acupuncture. In: Jayasuriya A (ed.). Medicina alternativa. Colombo: Sri Lanka Institute of Acupuncture; 1983. p. 1-36.

26 Bresser H. Allergietestung mit der Elektroakupunktur nach Dr. Voll. Allergologie 1992;14:364.
27 Bergold D. Elektroakupunktur nach Dr. Voll. ZFA (Stuttgart). 1976;6:312-22.

28 Lewith GT, Kenyon JN, Broomfield J, Prescott P, Goddard J, Holgate $\mathrm{S}$. Is electrodermal testing as effective as skin prick tests for diagnosing allergies? A double blind, randomised block design study. Br Med J 2001;322:131-4.

29 http://eavnet.com/index.html.

30 Wüthrich, B. Allergologie: Quo vadis? Schweiz Med Wochenschr 1998;129:905-14.

31 Young E, Patel S, Stoneham M, Rona R, Wilkinson JD. The prevalence of reaction to food additives in a survey population. J R Coll Physicians Lond 1987;21:241-7.

32 Friedman HM, Tortolani RE, Glick J, Burtis RT. Spelt is wheat. Allergy Proc 1994;15:240-5.

33 Nachrichten aus der Migros. Berichtigung: Brot für Weizenallergiker. Migros-Magazin 2005;29:21.

34 Stöger P, Wüthrich B. Type I allergy to cow milk proteins in adults. A retrospective study of 34 adult milk- and cheese-allergic patients. Int Arch Allergy Immunol 1993;102:399-407.

35 Wüthrich B. Neurodermitis und Rotationsdiät. Päd Praxis 1999;56:416-8.

36 Barrett S. www.quackwatch.org.

37 Ernst E. Bioresonance, a study of pseudo-scientific language. Forsch Komplementärmed Klass Naturheilkd 2004;11:171-3.

38 Angell M, Kassirer JP. Alternative medicine The risks of untested and unregulated remedies. N Engl J Med 1998;339:839-41.

39 Niggemann B, Grüber C. Side-effects of complementary and alternative medicine. Allergy 2003; 58:707-16.

40 Senna G, Passalacqua G, Crivellaro M, Bonadonna P, Gani F, Dorizzi R, et al. C. Unconventional medicine: a risk of undertreatment of allergic patients. Allergy 1999;54:1117-9.

41 Oepen I. Alternativmethoden in der Pneumologie aus rechtsmedizinischer Sicht. Atemw.-Lungenkrh 1995;21:30-6. 